\title{
Amitié, utilité, conquête ? Le statut culturel du français entre appropriation et rejet dans la Hollande prémoderne
}

Willem Frijhoff

\section{(2) OpenEdition}

1 Journals

Édition électronique

URL : https://journals.openedition.org/dhfles/3670

DOI : $10.4000 /$ dhfles.3670

ISSN : 2221-4038

Éditeur

Société Internationale pour l'Histoire du Français Langue Étrangère ou Seconde

Édition imprimée

Date de publication : 1 janvier 2013

Pagination : $29-48$

ISSN : 0992-7654

\section{Référence électronique}

Willem Frijhoff, « Amitié, utilité, conquête? Le statut culturel du français entre appropriation et rejet dans la Hollande prémoderne », Documents pour l'histoire du français langue étrangère ou seconde [En ligne], 50 | 2013, mis en ligne le 01 janvier 2017, consulté le 28 mars 2023. URL : http:// journals.openedition.org/dhfles/3670; DOI : https://doi.org/10.4000/dhfles.3670

Ce document a été généré automatiquement le 28 mars 2023.

Tous droits réservés 


\title{
Amitié, utilité, conquête ? Le statut culturel du français entre appropriation et rejet dans la Hollande prémoderne ${ }^{1}$
}

\author{
Willem Frijhoff
}

\section{Langue universelle, langue usuelle}

1 Dans son célèbre panégyrique de l'universalité de la langue française, écrit en 1782/83 et couronné un an plus tard, en 1784, par l'Académie de Berlin, Antoine de Rivarol (1753-1801) prit grand soin de marquer la supériorité du français sur l'anglais et l'allemand. Sage précaution aux yeux des Néerlandais de l'époque - dont d'ailleurs Rivarol ne fera la connaissance que bien plus tard, au cours de ses pérégrinations d'émigré en 1793/94, suite à la Révolution ${ }^{2}$. En effet, il rédige son traité au moment même où le français en tant que lingua franca se fait quasi insensiblement battre en brèche, en Europe septentrionale, par des langues concurrentes dans le domaine scientifique et littéraire ${ }^{3}$. Les littérateurs, philosophes, scientifiques et penseurs politiques allemands et anglais, de Goethe, Herder, Hegel, Schelling et un peu plus tard les Frères Grimm, à William Pitt, Jeremy Bentham, Jonathan Swift et Thomas Paine, sans oublier Benjamin Franklin, Thomas Jefferson ou Washington Irving outremer, faisaient puissamment concurrence à la popularité des écrits français ou francophones de la seconde moitié du XVIII ${ }^{e}$ siècle et du début du XIX ${ }^{e}$ siècle. En dépit des proclamations francophiles du roi de Prusse Frédéric le Grand et de la diffusion généralisée de l'usage du français dans la vie quotidienne des aristocraties cosmopolites de l'Europe, sa position comme langue de communication internationale de la bourgeoisie lettrée, conquise essentiellement sur le latin à partir de la seconde moitié $\mathrm{du} \mathrm{XVII}$ siècle, commençait à se faire grignoter sérieusement dans un nombre croissant de domaines. 
2 À la fin du XVIII ${ }^{e}$ siècle, les plans d'éducation des écoles primaires et secondaires dressés par les révolutionnaires néerlandais considéraient le français toujours comme une langue d'enseignement équivalente au néerlandais. À telle enseigne que le plan novateur de l'éducation secondaire, rédigé en 1792 par le recteur de collège Gerrit Vatebender (1759-1822) - avant même la Révolution batave de 1794/95 qui devait marquer pour deux décennies un renforcement structurel, mais politiquement fragile, de la position du français - ne prévoyait pas de cours spécifique de français, contrairement aux cours d'anglais, d'allemand, d'italien, de latin, de grec, voire d'hébreu (optionnel, il est vrai). Pour l'enseignant Vatebender, le français était la langue usuelle de l'école; on se l'appropriait dès l'école élémentaire, et on la perfectionnait au cours d'autres leçons, en particulier pendant les heures de dessin. Le citoyen révolutionnaire n'était-il pas naturellement bilingue franco-néerlandais ? ${ }^{4} \mathrm{Un}$ demi-siècle plus tard, le français a perdu son statut de langue quasi seconde pour devenir une langue étrangère comme les autres, la plus importante des langues modernes, certes, mais sans pouvoir maintenir son ancien statut privilégié. Il est significatif que la première grande école hollandaise de commerce, établie à Amsterdam en 1846 à l'initiative du médecin philanthrope Samuel Sarphati, enseignait pas moins de six langues étrangères, toutes à pied d'égalité. L'ordre reflète leur importance décroissante: français, allemand, anglais, italien, suédois et danois. Mais il n'est plus question d'enseignement en français. Désormais, le néerlandais sera la seule langue usuelle de l'enseignement - jusqu'à ce que l'anglais se profile comme la nouvelle lingua franca depuis les années 1970.

3 La prédominance que le français s'était acquise en Europe à la fin du XVIII ${ }^{e}$ siècle a inspiré toute une historiographie jubilatoire et universaliste. Sans y regarder de trop près, celle-ci a assimilé le modèle de Rivarol en postulant que par ses qualités imminentes - son ordonnance logique, et sa capacité de rendre les idées dites universelles, bref, en tant que langue de Descartes - le français était en quelque sorte promis de tout temps à son rôle prépondérant, et que seuls les malheurs de l'histoire avaient pu le détrôner par la suite. L'homme ne devrait-il pas se reconnaître naturellement bilingue franco-quelque-chose? Dans cet effort de synthèse je n'adopterai pas cette position jubilatoire, qui apparaît souvent d'une insupportable arrogance aux yeux des non-Français et fait plus de mal que de bien à la diffusion de la langue, mais celle du praticien indépendant et du consommateur critique. Au lieu d'interroger le français sur ses qualités linguistiques, je le scruterai sur sa valeur d'usage dans un contexte plus global, englobant les domaines culturel, social et politique ${ }^{5}$.

\section{Langue scientifique et langue vulgaire}

4 Dès la reprise ou, selon les cas, l'occupation des Pays-Bas septentrionaux par le pouvoir bourguignon au cours du XV $\mathrm{XV}^{\mathrm{e}}$ siècle, le français y prend une valeur considérable comme langue d'administration et de socialisation des élites. Un véritable bilinguisme politique et aristocratique s'y développe. Mais il ne faut pas commettre l'erreur de généraliser trop vite en mesurant un pays tout entier au comportement de ses élites, qu'elles soient politiques, administratives ou littéraires. C'est précisément par opposition à cette omniprésence du français dans les sphères du pouvoir que la bourgeoisie montante des $\mathrm{XVI}^{\mathrm{e}}$ et XVII ${ }^{\mathrm{e}}$ siècles, consciente de sa force dans le nouveau centre économique de 
l'Europe que constituaient les territoires de Flandre, de Brabant, de Zélande et de Hollande, formulait une contre-proposition linguistique: celle de la qualité du néerlandais comme langue de communication littéraire et scientifique, et de ses chances de devenir une nouvelle lingua franca.

5 Soulignons d'abord l'importance contemporaine de ce débat sur la langue scientifique ${ }^{6}$. Les nouvelles sciences cherchaient un nouvel idiome pour pouvoir s'exprimer adéquatement. Pour reprendre ici un argument courant aux XVI ${ }^{e}$ et XVII ${ }^{e}$ siècles : les Grecs ont inventé les sciences en parlant leur propre langue, le grec, et le latin était la langue maternelle des Romains - pourquoi donc nous-mêmes parlerions-nous en latin ${ }^{7}$ ? La renaissance des langues vulgaires et le premier essor du sentiment national un peu partout en Europe, aux $\mathrm{XV}^{\mathrm{e}}$ et $\mathrm{XVI}^{\mathrm{e}}$ siècles, avaient favorisé la codification des langues vulgaires et l'élaboration d'idiomes littéraires et scientifiques nationaux. En France, par exemple, Joachim du Bellay (1522-1560) se fait défenseur de l'emploi de la langue vulgaire, sans oublier la Dialectique française de Pierre de La Ramée (Ramus, 1515-1572) ${ }^{8}$.

6 Aux Pays-Bas, ce fut le Brabançon Johannes Goropius Becanus (1519-1572/73) qui dans ses Origines Antwerpianae afficha dès 1569 sa ferme conviction que le néerlandais [Diets, Duyts] était la langue la plus ancienne du monde, parlée au paradis par Adam et Ève mêmes". D'ailleurs, Diets était pour lui une contraction de "d'oudst ", c'est-à-dire "le plus ancien ». La prospérité ambiante des Pays-Bas, l'essor des sciences exactes, et la haute conjoncture des inventions techniques faisaient naître une demande linguistique que l'esprit de la Renaissance guidait en direction de la langue vulgaire. Ce n'est point le français international qui a assuré le développement de la prospérité en Hollande, ni même le latin, mais bien le néerlandais lui-même.

7 Dans la seconde moitié du XVI ${ }^{e}$ siècle et la première du XVII ${ }^{e}$ cet orgueil linguistique s'exprime dans la codification grammaticale du néerlandais par Hendrick Laurensz Spiegel (1584) et la publication des premiers dictionnaires par Van den Werve (1553) et Kiliaen (1574) dans le but avoué de créer une langue pure, refusant les mots étrangers ou bâtards empruntés au français ou au latin. Au même moment, l'ingénieur Simon Stevin (1548-1620) développe une argumentation scientifique sur l'excellence du néerlandais. Comme Becanus, il y voit un idiome très ancien, truffé de mots monosyllabiques qui étaient à leur tour faciles à réunir en mots composés utiles pour l'argumentation et la description scientifiques, sans oublier la capacité de la langue néerlandaise d'émouvoir, de passionner. Stevin crée lui-même un nouvel idiome mathématique en langue vulgaire (driehoek, vierkant, evenwijdig, etc.) qui de nos jours est toujours en usage. Rembertus Dodoens [Dodonaeus] en fait de même pour la botanique dans son Cruydt-Boeck ou Herbier (1554); son nouveau vocabulaire botanique s'adresse aux laïcs comme aux savants. Bien plus, Stevin aide à établir une académie d'ingénierie en langue néerlandaise, incorporée dans l'université de Leyde, qui allait permettre aux futurs ingénieurs de s'instruire dans la nouvelle langue scientifique qui serait aussi celle de leur travail quotidien. En somme, outre la renaissance du sentiment national et l'affirmation du sens civique, le retour aux langues vulgaires exprimait une volonté de domestication et de démocratisation des sciences et techniques qui ne pouvaient être assurées par les langues importées, français compris.

Ces efforts de néerlandisation des arts et des sciences ont rapidement buté sur le poids des institutions internationales, telles que les Églises et l'université, mais aussi sur l'inertie linguistique de la communauté scientifique nationale fermement vouée au 
latin avant de passer au français cultivé dans la République des Lettres. Ainsi, les immigrés francophones ont rapidement réussi à imposer à l'Église Réformée un Synode autonome, l'Église wallonne. Quant aux universités, il y avait bien en permanence quelques maitres de français dans leur orbite, le plus célèbre et influent étant sans doute Jean-Nicolas de Parival (1605-1669) avec son très populaire Délices de la Hollande $(1651)^{10}$, mais seule l'université frisonne de Franeker ouvrira un siècle plus tard, en 1754, une véritable chaire de français. Significativement, toutefois, le professeur choisi, Jacques Garcin (1720-1796), un Genevois, ancien précepteur et pasteur wallon, prononça sa leçon inaugurale De utilitate linguae gallicae en latin (1757) ${ }^{11}$. C'est que sa chaire représentait et assurait l'autre filière d'acquisition du français, encore peu étudié, les élites empruntant la voie du latin classique pour apprendre le français, par opposition aux classes moyennes qui apprenaient le français par le truchement de leur langue maternelle, sous la direction de maîtres de langue ou de manuels d'apprentissage. Devant l'évident déclin du latin, Garcin souligne avec ardeur l'utilité $\mathrm{du}$ français et son usage universel dans la vie sociale, commerciale, diplomatique et scientifique. Au fur et à mesure que la Hollande affirmait sa place en Europe et développait son réseau international de lettrés, de libraires et de savants, on y montrait effectivement une nette tendance à délaisser le néerlandais au profit de l'une ou l'autre lingua franca universitaire: d'abord le latin, puis, depuis la seconde moitié du XVII ${ }^{\mathrm{e}}$ siècle le français en tant que langue de publication ou de conversation des universitaires, concurrencé vers la fin du XVIII ${ }^{\mathrm{e}}$ siècle par l'allemand, dont l'importance et la diffusion montent en médecine, dans les sciences exactes, dans les lettres et dans les nouvelles sciences humaines, telle que l'ethnologie et l'ethnographie (ou folklore), sciences décisives pour le discours sur l'identité nationale et que la France découvrira sous la Révolution ${ }^{12}$.

9 En fait, la résistance du latin comme langue universitaire est assez remarquable ${ }^{13}$. Le français n'a jamais réussi à s'établir en Hollande comme langue d'enseignement universitaire, malgré sa large diffusion dans les élites cultivées, et en dépit du fait que les professeurs et étudiants eux-mêmes écrivaient une partie de leur correspondance en français. Outre un épiphénomène de la vivante tradition humaniste, cette longévité du latin traduit aussi un changement dans son statut éducatif. Vénéré aux $\mathrm{XVI}^{\mathrm{e}}$ et XVII ${ }^{\mathrm{e}}$ siècles comme la langue d'imitation des grands exemples de l'Antiquité, au XVIII le latin, tout comme le grec, passait à un niveau supérieur, moral. Apprécié comme le véhicule idéal des valeurs humanistes que la jeunesse devait assimiler, au XIX ${ }^{e}$ le latin était considéré comme l'instrument privilégié de la Bildung, c'est-à-dire de la haute culture qui forme l'esprit sans présenter une valeur utilitaire immédiate. Il devint donc le rempart éprouvé contre les broodstudiën [les études strictement professionnelles, sans intérêt intellectuel] et contre les nouveautés venant du Sud, entendez la France agnostique, révolutionnaire ou laïque, dont justement la valeur utilitaire était alors fortement accentuée.

10 Tout ceci explique pourquoi le XVIII ${ }^{\mathrm{e}}$ siècle, tout en étant le grand siècle de l'influence française en Europe, ne permettra pas vraiment à la langue française de s'imposer comme la langue scientifique, la lingua franca de l'université. Un ouvrage publié chez Georges Gallet à Amsterdam au tournant du siècle, La rhétorique de l'honnête homme, ou la manière de bien écrire des lettres, de faire toutes sortes de discours, etc. (1700), indique bien le terrain d'usage principalement couvert par la langue française. Elle demeure, en fait, cantonnée dans ses domaines traditionnels : la vie quotidienne des élites, la civilisation de cour et le savoir-vivre, le beau langage, la littérature et l'esthétique, le commerce, le 
tourisme, et la religion des immigrés. Le XVIII ${ }^{e}$ siècle hollandais connaît en fait un régime trilingue. Pour emprunter les paroles qu'une mère aristocrate, Carolina van Haren, adresse alors à son fils Gijsbert Karel van Hogendorp, le futur homme d'État fondateur du royaume orangiste : on utilise le latin pour les livres, le français pour la politesse et la correspondance, le néerlandais pour la conversation ${ }^{14}$.

\section{Langue commerciale, langue sociale}

11 Si le français n'a pas réussi à se tailler la part du lion dans la vie intellectuelle des PaysBas, il ne faut pas sous-estimer pour autant son importance dans la formation du jeune Néerlandais d'Ancien Régime. En fait, il importe de distinguer ici entre le français en tant que langue d'usage quotidien, donc de socialisation élémentaire, et en tant que langue de communication internationale, la lingua franca. Le français constitue assez tôt la langue seconde ou même première de plusieurs groupes d'habitants des ProvincesUnies qui l'apprennent par leurs parents, à l'école dite française, avec un maître de langue ou par autodidaxie dans un manuel - tels les réfugiés wallons ou huguenots, les élites cultivées autochtones, les marchands étrangers, la librairie et les intellectuels immigrés - mais ce n'est qu'à la fin du XVII e siècle que le latin commence vraiment à céder du terrain au français comme langue de communication internationale de la République des Lettres, tout en continuant de se réserver jalousement l'université.

12 Le français usuel des réfugiés reposait sur l'utilité quotidienne de la langue comme symbole et instrument de l'identité du groupe. Au fur et à mesure de leur assimilation, ce français a perdu du terrain dans la communauté globale - malgré l'extension simultanée du français comme lingua franca internationale au sein de la société. Mais il faut bien reconnaître que nous ne savons pas grand-chose de la diffusion réelle et de l'usage concret du français dans la vie quotidienne des Néerlandais d'autrefois. Nous savons un peu de l'offre, mais beaucoup moins de la demande et très peu de la pratique de la langue. Nous connaissons le nombre approximatif de manuels, méthodes, grammaires et dictionnaires français parus en Hollande sous l'Ancien Régime - sans doute quelque 300 éditions au bas mot - et l'existence de nombreuses écoles dites françaises, c'est-à-dire des écoles de commerce et d'arts utiles où le français était la langue usuelle, au moins une dans presque chaque ville ou gros bourg du pays ${ }^{15}$.

Il est vraisemblable - à preuve les remarques aigres-douces de leurs interlocuteurs français - que le français des Hollandais fût presque toujours assez lourd et approximatif, plutôt colloquial que littéraire. Mais nous savons aussi qu'outre les écoles proprement françaises un grand nombre d'écoles élémentaires était dirigé par un maitre sachant un minimum de français et s'appliquant à le transmettre lorsque l'occasion se présentait. L'enquête scolaire de 1811 nous apprend ainsi que $15 \%$ des écoles élémentaires étaient tenues par un maître parlant français, les deux tiers des écoles ou pensionnats du niveau secondaire étant de langue française ${ }^{16}$. Il est probable que ce pourcentage était nettement plus élevé au XVII ${ }^{e}$ siècle, du moins dans les villes des provinces côtières et commerçantes, où un très grand nombre de maîtres d'école réfugiés des provinces méridionales tenues par le roi d'Espagne s'était établi ${ }^{17}$.

Les titres mêmes des ouvrages utilisés pour l'enseignement du français au début du $\mathrm{XVII}{ }^{\mathrm{e}}$ siècle indiquent l'objectif de ces écoles et de l'enseignement des maitres de langue: De l'office d'une bonne matronne de Gabriel Meurier, La fontaine d'honneur et de vertu, le Miroir des mesnageres, le Miroir des vrayes meres, et le Miroir des vefves, trois 
pièces de théâtre scolaire de l'ex-Anversois Peter Heyns, mais aussi plusieurs volumes ou «trésors » de dialogues, de lettres, de propos, de proverbes ou de sentences - donc des manuels ou des aides à la conversation -, dont l'un portait le titre attendrissant $L e$ Perroquet mignon des petits enfants françois-flameng, sans même parler du petit manuel bilingue de Magdaleine Valery, maîtresse d'école pour les filles à Leyde, La Montaigne des Pucelles, en neuf dialogues, sur les noms des neuf Muses, contenant diverses belles \& vertueuses Doctrines, à l'instruction de la jeunesse (1599). Dès 1610, le catalogue de vente de Cornelis Claesz, le plus grand libraire d'Amsterdam, recense 73 différents titres scolaires de langue française ${ }^{18}$. Le français colloquial y domine, mais il est doublé de deux autres fonctions qui le soutiennent socialement : langue du commerce, la France étant toujours le partenaire commercial principal de la Hollande, et langue de socialisation morale et esthétique ${ }^{19}$.

En ce début du XVII ${ }^{e}$ siècle, l'usage du français, tout en étant assez largement répandu, ne renvoie pas encore à une réception généralisée de cette langue en Hollande. Il s'agit soit d'une diglossie lapidaire mais localement limitée, nécessaire dans un pays où les réfugiés francophones sont parfois si nombreux qu'ils tendent à former des communautés autonomes à l'intérieur des villes (ainsi à Leyde, Middelbourg ou Rotterdam); soit d'une langue d'usage limitée, liée à des groupes, des circonstances ou des situations sociales précises : le commerce, les arts et techniques, la socialisation des élites. Le français n'est point alors la langue de l'éloquence, ce qui demeurera encore longtemps l'apanage du latin ou du néerlandais lui-même. Il n'est même pas encore la langue des savants, quand bien même des savants écrivent en français. Bien au contraire, l'on se bat, dans les cercles universitaires français, pour obtenir du célèbre savant français Joseph Juste Scaliger, philologue et historien né en France mais établi à Leyde, un billet autographe rédigé dans son latin classique, d'une perfection et d'une clarté inimitables. Les bibliothèques savantes dont nous avons conservé l'inventaire confirment cette impression: au tournant du $\mathrm{XVI}^{\mathrm{e}}$ siècle vingt grandes collections privées hollandaises totalisent 20.000 livres dont $80 \%$ en latin contre 9,3\% en français (van Selm 1987 : 115). C'est seulement vers la fin du XVII ${ }^{e}$ siècle que le français prend plus d'importance comme langue scientifique. La rubrique des comptes rendus dans l'important périodique savant allemand Acta eruditorum reflète le niveau linguistique de la production savante en Europe : la francisation de la science double en un siècle, elle atteint $18 \%$ dans les premières années du XVIII ${ }^{e}$ siècle, avec un forte pointe en Hollande $^{20}$. Elle va au détriment du latin qui perd, en outre, des points sur l'anglais. L'anglais ne totalise encore que $8 \%$ mais augmente lentement, tout comme la part de l'allemand. L'on ne saurait donc négliger ce nouveau venu en se polarisant indûment sur l'omniprésence du français. Mais à ce tournant que Paul Hazard a jadis appelé la crise de la conscience européenne, la communication scientifique internationale continue malgré tout de se faire essentiellement en latin.

Jusque-là, l'anglais était utilisé essentiellement dans les relations commerciales avec la Grande-Bretagne et, passivement, dans la littérature puritaine que les calvinistes néerlandais assimilaient avidement dès le début du XVII ${ }^{e}$ siècle. Mais au XVIII ${ }^{e}$ siècle l'anglais commence à conquérir l'Europe par un double biais, laissé en friche autant par le latin que par le français : d'une part la littérature moralisatrice et politique, d'autre part les sciences exactes et les techniques. Le discours politique et le débat public dans un espace libre naissent en Angleterre, non pas en France, où la censure royale pouvait être féroce et où la Révolution transforme ce débat presque immédiatement en un discours partisan. Dans la seconde moitié du XVIII ${ }^{\mathrm{e}}$ siècle, l'allemand fait une entrée 
remarquée sur la scène linguistique néerlandaise. Il conquiert une partie du marché littéraire et philosophique où il fait reculer la part de la littérature française ${ }^{21}$. Mais grâce à l'excellence de la science allemande, il commence aussi à dominer le marché du livre médical, scientifique, ethnographique, sans parler de la théologie et de la spiritualité piétiste, omniprésente dans le Nord de l'Europe. On comprend dès lors beaucoup mieux pourquoi Rivarol dans son discours berlinois sur l'universalité de la langue française se soit donné tant de peine à exalter la prééminence du français sur l'anglais et l'allemand : c'étaient là, en effet, les deux grandes rivales du moment de la langue française, et elles devaient le rester.

Il faut donc relativiser, et surtout replacer avec précision sur une échelle chronologique, la place et l'usage du français dans la société hollandaise globale. Il n'en demeure pas moins qu'à l'apogée de l'influence française la connaissance du français a pu paraitre universelle dans la République néerlandaise aux yeux des observateurs étrangers. Dans tous les milieux qu'ils touchaient, le français était une langue facile d'accès. Cette expérience a pu renforcer à son tour le sentiment d'universalité de la langue elle-même, comme en témoigne l'approche de Rivarol qui ne néglige point le facteur de son usage. Je ne citerai qu'un seul témoignage de ce sentiment. Dans son État présent de la République des Provinces-Unies, paru en 1729-30, François-Michel Janiçon affirme d'emblée que «la Langue Françoise y est aussi fort en usage, par le grand nombre de François Protestants qui s'y sont réfugiez, depuis la Révocation de l'Édit de Nantes en 1685. Et, comme toutes les Négociations entre la République et les autres Puissances de l'Europe se font presque toutes aujourd'hui en François, il n'y a point de Membre de la Régence, qui ne se pique de savoir cette Langue, et de la faire apprendre à ses Enfans. Les Négocians et les autres Bourgeois ont la même ambition ${ }^{22}$. Janiçon note donc la généralisation du français par un double biais : politique - ou mieux, politico-religieux - et commercial. Alors qu'il fait remonter la première à la Révocation et, sans le dire expressément, aux négociations de Nimègue qui vers 1678 avaient inauguré la prédominance du français comme langue diplomatique, le français de la pratique commerciale et de la culture bourgeoise remonte bien plus haut.

\section{Critiques bataves}

Dès le début du XVII ${ }^{e}$ siècle, cependant, les puristes hollandais étaient partis en guerre contre la francisation rampante du pays, venue dans le sillage des réfugiés wallons qui, soulignons-le, n'étaient pas des Français mais des Brabançons, Flamands, Hennuyers, Liégeois ${ }^{23}$. Outre leur fortune et leur langue, souvent fortement teintée de dialectes locaux mais toujours opposée au néerlandais, ceux-ci avaient apporté des manières précieuses, des modes vestimentaires et un goût du luxe qui tranchaient sur la sobriété batave antique dont les Hollandais eux-mêmes se targuaient volontiers ${ }^{24}$. Dans l'image que les Hollandais donnaient de ces usurpateurs de leur identité, le thème de la domination par le francophone conquérant et arrogant revient fréquemment. Suite à la guerre de Hollande et à la politique religieuse du Roi Soleil, cette critique passe du francophone indifférencié, qu'il soit Brabançon, Bourguignon, Savoyard, Suisse ou Français, aux membres de la nation française en se réduisant insensiblement à la France elle-même en tant qu'unité politique. L'extension de l'usage de la langue française sur un nombre croissant de domaines de la vie sociale est alors associée au 
danger politique et moral que la nation française représente pour la survie de la nation néerlandaise qui est encore à la recherche de son identité ${ }^{25}$.

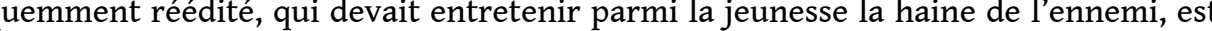
après 1672 appliqué aux Français. La Tyrannie française,illustrée des gravures suggestives de Romeyn de Hooghe sur les atrocités perpétrées, dénonce la perfidie française à l'égard des protestants lors du massacre de la Saint-Barthélemy (1572) et les cruautés similaires commises en 1672 par l'armée française dans deux villages à la limite de la province de Hollande, Bodegraven et Zwammerdam. La Révocation et ses dragonnades, puis la répression religieuse dans les Cévennes s'y ajouteront bientôt. Pendant plus d'un siècle, jusqu'à ce que les Anglais prennent le relais dans les années 1780, ces images cruelles forment la toile de fond nécessaire à la réputation du Français comme nouvel ennemi national, aussi bien parmi la moyenne et petite bourgeoisie qu'au sein du petit peuple qui, essentiellement de tendance orangiste, n'a jamais été franchement francophile ${ }^{27}$. Devant les horreurs de la Révolution, cependant, le sentiment anti-français redouble de vigueur. Dès 1793 trois Tyrannies paraissent : une Tyrannie de la faction jacobine, en France ${ }^{28}$, La tyrannie française, ou le théâtre du massacre des années 1792 et $1793^{29}$, et un nouveau Miroir de la jeunesse, ou Nouvelle Tyrannie française ${ }^{30}$. Dans ce dernier, en forme de dialogue éducatif, le père commence par rappeler à son fils le vieux stock de ressentiments, la Saint-Barthélemy et l'invasion de 1672, pour enchaîner sur la Révolution française. Et le fils de répondre qu'il comprend maintenant pourquoi son père « parle toujours avec tant de mépris de la nation française et rejette toute allusion à leur savoir-vivre, leur politesse, leur bonnes manières ».

21 Tout bien pesé, il peut paraître étonnant que la langue française ait joui d'une si grande fortune dans un pays qui a tant détesté la France elle-même ou ses habitants. On y verra une conséquence de la liberté d'opinion et de presse qui régnait en Hollande. Même au plus fort de la guerre, une discussion relativement libre demeurait possible. Dans l'année même où, à la suite de l'invasion de 1672, la presse hollandaise honnit et vomit tout ce qui sent le Français, le célèbre éditeur Joan Blaeu publie à Amsterdam une traduction du Nouveau Traité de la civilité qui se pratique en France, parmi les honnestes gens (1671) d'Antoine de Courtin, qui venait d'être publié à Paris, puis à Amsterdam même ${ }^{31}$. Ce traité devait durablement marquer le comportement social des élites hollandaises.

Autre exemple de cette liberté de penser; Franciscus Ridderus, un influent pasteur réformé de Rotterdam, s'efforça dès 1674, en pleine guerre, de promouvoir une image plus juste et plus nuancée des différents ennemis, dans une série de portraits dialogués appelés Le Français historique, L'Anglais historique, L'Espagnol historique, et Le Hollandais historique. Ces dialogues montrent l'ambiguïté des relations franco-hollandaises. Force d'attraction d'une part, méfiance ou mépris de l'autre. Dans tous les cas, la francisation, 
appelée ou honnie, concerne autant la langue, véhicule principal de la culture française, que les modalités mêmes de cette culture, et plus particulièrement les modèles sociaux qu'elle entendait promouvoir. Ainsi, l'honnête homme, image positive chérie par l'aristocratie et le patriciat, peut dans une autre lecture prendre la figure d'un homme arrogant, libertin, ou courtisan, ridicule dans ses prétentions et facilement démasqué par ces solides gaillards que sont les Hollandais dans leur propre perception : ces Français, ce n'est rien que du vent ${ }^{32}$.

Mais la capitulation linguistique et morale devant ces mêmes Français dès avant la guerre de Hollande a aveuglé et affaibli les élites dirigeantes. Aux yeux des critiques, elle a préparé de l'intérieur la victoire du Roi Soleil. Tout au long du XVIII ${ }^{\mathrm{e}}$ siècle, cette image complexe, où langue et arts, modes et mœurs, admiration et crainte se mêlent inextricablement et où résonne également une sourde critique sociale contre l'attitude traitresse des élites autochtones, l'ennemi intérieur, sera mise en avant par les censeurs du déclin national, en particulier dans les écrits spectatoriaux. Les caprices du maniérisme français, estimé superficiel et à la poursuite de la mode du jour, l'arrogance, l'esprit hiérarchique, les arguties et les chicanes, le libertinage et le relâchement des mœurs, le tout symbolisé dans l'adoption de la langue française ou dans l'abâtardissement du néerlandais truffé de mots et expressions empruntés au français, sont tenus pour responsables de toutes les déviances morales de la société hollandaise $\mathrm{e}^{33}$. Le retour à la pureté originale du peuple passera donc par l'élimination des corruptions françaises. On comprend dès lors que les patriotes bataves de la fin du $\mathrm{XVIII}$ siècle avaient à mener un difficile débat intérieur. Tout en prenant exemple sur la Révolution française, ils devaient en même temps repousser l'accusation de connivence avec l'ennemi moral de toujours. Les relations ne pouvaient donc qu'être extrêmement complexes entre les Bataves, qui appelaient les Français comme libérateurs, et les Français eux-mêmes, qui se comportaient comme envahisseurs exactement comme l'image des manuels scolaires les avait dépeints depuis plus d'un siècle.

Pour conclure, j'en fournirai ici un exemple éloquent. Dans son numéro du 17 juin 1805, une lettre non signée, apparemment envoyée par un Hollandais de La Haye, parut dans Le Moniteur universel, journal officiel de l'Empire. Il entendait sonner le glas de la langue néerlandaise. «La langue française, affirme l'auteur, devient de jour en jour d'un usage plus habituel dans ce pays-ci. La plupart des Hollandais distingués par leur fortune ou leur éducation, la parlent entr'eux dans l'intérieur de leurs familles, de préférence à la langue du pays ; de sorte que celle-ci s'en va tout doucement se reléguer dans les basses classes du peuple; et il n'en restera peut-être, dans cinquante ans, qu'un patois que les gens comme il faut abandonneront à leurs domestiques, aux ouvriers et aux matelots ${ }^{34} »$.

L'article provoque un tollé parmi les dirigeants politiques et les intellectuels qui se retournent massivement contre les prétentions du français en tant que véhicule politique de l'idéologie impériale. Le Comité national [Groot besogne] qui en mars 1806 délibère fébrilement sur les éléments d'identité nationale à préserver à tout prix devant l'annexion menaçante, y inclut d'emblée le maintien de la moedertaal, la langue maternelle. On ne va certes pas jusqu'à rejeter l'emploi du français lui-même dans la vie quotidienne des Pays-Bas. Bien au contraire, en affirmant que depuis bien longtemps le français est parlé dans un grand nombre de ménages, on approuve cet usage. «L'exercice de la langue française sera toujours très utile, sinon indispensable, mais il 
ne faut point négliger pour autant notre propre belle langue ». Mais si le français demeure simplement utile, le néerlandais est paré de cette esthétique originelle qui est instillée à chaque citoyen, comme une marque d'identité, au sein maternel ${ }^{35}$. Une fois de plus, nous retrouvons donc ici le lien fort et étroit entre le sort de la langue et l'identité collective. Les Néerlandais adoptent le français comme lingua franca, la langue qui est pour eux à ce moment la plus utile internationalement, mais ils refusent l'idéologie de supériorité politique, socio-culturelle ou esthétique qui sous-tend le concept d'universalité linguistique.

\section{NOTES}

1. Ce texte reprend et résume différents travaux que j'ai présentés au cours des années, en particulier: (1996). «Le Français en Hollande après la Paix de Westphalie : langue d'immigrés, langue d'envahisseurs, ou langue universelle?». Documents pour l'histoire du français langue étrangère ou seconde, no 18, 329-350 ; (1998). «Des origines à 1780 : l'émergence d'une image ». In Willem FRIJHOFF \& André REBOULLET (dir.). Histoire de la diffusion et de l'enseignement du français dans le monde [Numéro spécial de: Le français dans le monde: Recherches et applications]. Paris: Hachette/Edicef, 8-19 ; (2010). Meertaligheid in de Gouden eeuw : Een verkenning. KNAW, Mededelingen van Afdeling Letterkunde, NR 73, no 2. Amsterdam: KNAW Press. En ligne: <www.knaw.nl/ publicaties/pdf/20101019/pdf>.

2. RIVAROL (1785). De l'universalité de la langue française. Discours qui a remporté le prix à l'Académie de Berlin en 1784. $2^{\mathrm{e}}$ éd. Berlin \& Paris : Prault \& Bailly, 85 ; RIVAROL (1930). De l'universalité de la langue française. Texte établi et commenté par Th. Suran. Paris \& Toulouse $: H$. Didier, 60-64.

3. Voir pour l'anglais aux Pays-Bas : P.L.M. LOONEN (1991). « For to learne to buye and sell »Learning English in the Low Dutch area between 1500 and 1800. A critical survey. Amsterdam \& Maarssen : APAHolland University Press. Longtemps la connaissance passive de l'anglais était beaucoup plus grande que sa maîtrise active, qui ne se développera qu'à la fin du XVIII ${ }^{\mathrm{e}}$ siècle. Voir plus généralement: Th.J.M. van ELS \& M.F. KNOPS (1988). « The history of the teaching of foreign languages in the Low Countries ». Historiographia linguistica, XV/1-2, 289-316; Jan NOORDEGRAAF \& Frank VONK (dir.) (1993). Five Hundred Years of Foreign Language Teaching in the Netherlands 1450-1950. Amsterdam: Stichting Neerlandistiek VU/Münster: Nodus.

4. G.C.C. VATEBENDER (1792). «Plan van een Nederlands Opvoedings-school ». Mengelwerken der Kamer van Rhetorica, genaemd De Goudsbloemen, 21-136; voir à son sujet également, Willem FRIJHOFF (1997). «Valeurs militaires, élites civiles, sciences exactes: projets de réforme de l'éducation secondaire en Hollande à l'époque révolutionnaire ». In Jean-Paul BERTAUD, Françoise BRUNEL, Catherine DUPRAT \& François HINCKER (dir.), Sur la Révolution: approches plurielles. Mélanges Michel Vovelle. Paris : Société des études robespierristes, 405-414.

5. Sur la question, la méthode et le pays concerné : Willem FRIJHOFF (1988). « Langues nationales, langues de contact, langues de culture ». In Europe sans rivage. Symposium international sur l'identité culturelle européenne, Paris, janvier 1988. Paris : Albin Michel, 76-83 ; (1990). «L'usage du français en Hollande, XVII ${ }^{\mathrm{e}}$-XIX ${ }^{\mathrm{e}}$ siècles: propositions pour un modèle d'interprétation ». Études de linguistique appliquée, 78, 17-26; (1991). «Le plurilinguisme des élites en Europe de l'Ancien Régime au début du XX $\mathrm{X}^{\mathrm{e}}$ siècle ». In Daniel COSTE \& Jean HÉBRARD(dir.). Vers le plurilinguisme des élites. Numéro thématique de Le français dans le monde. Recherches et applications, 120-129; (1995). 
«La formation des négociants de la République hollandaise ». In Franco ANGIOLINI \& Daniel ROCHE (dir.). Cultures et formations négociantes dans l'Europe moderne. Paris : EHESS, 175-198.

6. G.R.W. DIBBETS (1992). « Dutch philology in the $16^{\text {th }}$ and $17^{\text {th }}$ century ». In J. NOORDEGRAAF, K. VERSTEEGH \& K. KOERNER (dir.). The History of Linguistics in the Low Countries. Amsterdam \& Philadelphie : Rodopi, 39-61; Marijke van der WAL (1995). De moedertaal centraal. Standaardisatieaspecten in de Nederlanden omstreeks 1650. La Haye: SDU Uitgevers, 23-34; Marijke van der WAL \& Cor van BREE [1992] (2008). Geschiedenis van het Nederlands. $5^{\mathrm{e}}$ éd. Houten: Het Spectrum, 183-199. Sur la lingua adamica : Umberto ECO (1993). Ricerca della lingua perfetta nella cultura europea. Rome \& Bari : Laterza. Trad. fr. (1994) La recherche de la langue parfaite dans la culture européenne. Paris : Seuil ; Allison P. COUDERT (dir.) (1999). The Language of Adam/Die Sprache Adams. Wiesbaden: Harrassowitz. Sur les langues scientifiques de l'époque moderne: Roger CHARTIER \& Pietro CORSI (dir.) (1996). Sciences et langues en Europe. Paris : EHESS.

7. L. Van den BRANDEN [1956] (1967). Het streven naar verheerlijking, zuivering en opbouw van het Nederlands in de $16^{\text {de }}$ eeuw. Gand: Kon. Vlaamse Akademie voor Taal- en Letterkunde. repr. Arnhem : Gijsbers \& van Loon.

8. K. van BERKEL (1987). "Ramus, précurseur de Descartes aux Pays-Bas ». Septentrion, Revue de culture néerlandaise, $16: 2$, 30-36; Howard HOTSON (2007). Commonplace Learning: Ramism and its German Ramifications, 1543-1630. Oxford: Oxford University Press.

9. Marijke van der WAL (1995). «Early language typology. Attitudes towards languages in the $16^{\text {th }}$ and $17^{\text {th }}$ centuries $»$. In K.D. DUTZ \& K.A. FORSGREN (dir.). History and rationality. Münster : Nodus, 93-106 ; Tom DENEIRE \& Toon van HAL (2006). Lipsius tegen Becanus: over het Nederlands als oertaal. Editie, vertaling en interpretatie van zijn brief aan Hendrik Schotti (19 december 1598). Amersfoort : Florivallis.

10. Andreas NIJENHUIS (2009). « La Hollande du Siècle d'Or à l'aune des Délices de la Hollande de Jean-Nicolas de Parival ». In Gilles BERTRAND (dir.). Voyage et représentations réciproques (XVI ${ }^{e}$-XIX ${ }^{e}$ siècle). Méthode, bilans et perspectives. Grenoble : CRHIPA, 29-48; également dans sa thèse (2012), Les "Voyages de Hollande ». La perception française des Provinces-Unies dans la première moitié du XVII siècle. Thèse de doctorat. Amsterdam : Université Libre, 327-351.

11. Jacobus GARCIN (1757). Oratio inauguralis de utilitate linguae gallicae. Franeker: G. Coulon. [Bibl. Univ. Amsterdam : OBR. Fol. 640]. Sur lui : Nieuw Nederlandsch Biografisch Woordenboek, IV (1918). Leyde. col. 630 ; K.-J. RIEMENS (1919). Esquisse historique de l'enseignement du français en Hollande du XVI $I^{e}$ au XIX ${ }^{e}$ siècle. Leyde : Sijthoff, 157, 187-188.

12. Ferdinand van INGEN (1981). Holländisch-deutsche Wechselbeziehungen in der Literatur des 17. Jahrhunderts. Bonn: Presse- und Kulturabteilung der Kgl. Niederländischen Botschaft; Guillaume van GEMERT \& Dieter GEUENICH (dir.) (2003). Gegenseitigkeiten. Deutsch-niederländische Wechselbeziehungen von der frühen Neuzeit bis zur Gegenwart. Essen : Pomp Verlag.

13. Françoise WAQUET (1998). Le latin, ou l'empire d'un signe, $X V I^{e}-X X^{e}$ siècle. Paris : Albin Michel.

14. Henriëtte L.T. de BEAUFORT (1979). Gijsbert Karel van Hogendorp, grondlegger van het koninkrijk. $4^{\mathrm{e}}$ éd. revue. Rotterdam: Donker, 37. Cf. à ce sujet Willem FRIJHOFF (1991).

15. L'état de la question dressé par K.-J. RIEMENS ( $c f$. note 11) n'a pas encore été remplacé, en dépit des progrès de la recherche. D’utiles compléments dans R. MURRIS (1925). La Hollande et les Hollandais aux XVII et XVIII ${ }^{e}$ siècles vus par les Français. Paris : E. Champion ; Christiane BERKVENSSTEVELINCK (1985). « De Hugenoten ». In P. BLOM, et al. (dir.). La France aux Pays-Bas. Invloeden in het verleden. Vianen : Kwadraat, 13-49; Willem FRIJHOFF (1986). « Modèles éducatifs et circulation des hommes : les ambiguïtés du second Refuge ». In La Révocation de l'Édit de Nantes et les ProvincesUnies, 1685. Colloque international du Tricentenaire, Leyde, 1-3 avril 1985. Amsterdam \& Maarssen: APA-Holland University Press, 51-75, ainsi que dans les nombreuses publications de MarieChristine KOK ESCALLE et Madeleine van STRIEN-CHARDONNEAU qui préparent actuellement une nouvelle synthèse. Voir en attendant, Willem FRIJHOFF (1983). "Van onderwijs naar opvoedend onderwijs. Ontwikkelingslijnen van opvoeding en onderwijs in Noord-Nederland in de 
achttiende eeuw ». Werkgroep Achttiende Eeuw, Onderwijs en opvoeding in de achttiende eeuw. Amsterdam \& Maarssen: Holland University Press, 3-39; Nan L. DODDE \& Cees ESSEBOOM (2000). «Instruction and Education in French Schools. A Reconnaissance in the Northern Netherlands 1550-1700 ». In Jan de CLERCQ, Nico LIOCE \& Pierre SWIGGERS (dir.). Grammaire et enseignement du français, 1500-1700. Louvain : Peeters, 39-60.

16. Willem FRIJHOFF (1986). «Université et marché de l'emploi dans la République des Provinces-Unies ». In Dominique JULIA, Jacques REVEL et Roger CHARTIER (dir.). Les Universités européennes du XVIe au XVIII ${ }^{e}$ siècle: Histoire sociale des populations étudiantes, I. Paris: EHESS, 205-243, 223, tableau 4.

17. Jan BRIELS (1976). De Zuidnederlandse immigratie in Amsterdam en Haarlem omstreeks 1572-1630 (thèse de doctorat, Utrecht, 1976; J.G.C.A. BRIELS (1972, 1973). "Zuidnederlandse onderwijskrachten in Noordnederland 1570-1630». Archief voor de geschiedenis van de katholieke kerk in Nederland, 14, 89-169, 277-298; 15, 103-149, 263-297; J. BRIELS (1985). Zuidnederlanders in de Republiek 1572-1630: Een demografische en cultuurhistorische studie. Sint-Niklaas, B.: Danthe;Willem FRIJHOFF (1998). « Migrations religieuses dans les Provinces-Unies avant le second Refuge ». Revue du Nord, 80, n 326-327, 573-598.

18. Bert van SELM (1987). Een menighte treffelijcke Boecken. Nederlandse boekhandelscatalogi in het begin van de zeventiende eeuw. Utrecht: HES, 234-242, 275-285, en donne la liste complète avec identifications. Voir aussi A. Hendrickx (1961). « Franse school- en leerboeken in de $16^{\mathrm{e}}$ en de $17^{\mathrm{e}}$ eeuw ». Paedagogica historica, 1, 225-243.

19. Sur les maîtres de langue française : Marie-Christine KOK ESCALLE \& Madeleine van STRIENCHARDONNEAU (2005). "Apprentissage de la langue et comparatisme culturel en Hollande : le métier de maître de langue (XVII ${ }^{\mathrm{e}}$-XIX ${ }^{\mathrm{e}}$ siècles)». Documents pour l'histoire du français langue étrangère ou seconde, 33-34, 120-143; des mêmes, «Van Parival tot Baudet. De Franse taalmeester en zijn leerlingen in de Noordelijke Nederlanden, van de $17^{\mathrm{e}}$ tot de $19^{\mathrm{e}}$ eeuw. Vorming tot cultureel comparatisme via het vreemde-talenonderwijs ", in: e-meesterwerk. E-tijdschrift van het PHG [Peeter Heyns Genootschap], publié le 4 mars 2009. En ligne: $<$ www.peeterheynsgenootschap.nl/e-meesterwerk.html>.

20. H. LAEVEN \& G. van GEMERT (1982). «De Acta Eruditorum als invalshoek voor de Noordnederlandse boekproductie (1682-1720)». Documentatieblad Werkgroep Achttiende Eeuw, no 53-54, 85-117.

21. A. NIEUWEBOER (1982). «De populariteit van het vertaalde verhalend proza in $18^{\mathrm{e}}$-eeuws Nederland, en de rol van de boekhandel bij de praktijk van het vertalen ».Documentatieblad Werkgroep Achttiende Eeuw, no 53-54, 119-141.

22. François-Michel JANIÇON (1729-1730). État présent de la République des Provinces-Unies. 2 vol. La Haye : Van Duren, I, 12-13 ; Madeleine van STRIEN-CHARDONNEAU (1994). Le Voyage de Hollande : récits de voyageurs français dans les Provinces-Unies, 1748-1795. Oxford : Voltaire Foundation, 142-144. 23. Sur le problème de la francisation: Willem FRIJHOFF (1989). «Verfransing? Franse taal en Nederlandse cultuur tot in de Revolutietijd». Bijdragen en mededelingen betreffende geschiedenis der Nederlanden, 104, 592-609.

24. Pour l'importance, les avatars et les fonctions du mythe batave dans le développement de la conscience nationale néerlandaise, voir Willem FRIJHOFF (1994). «L'évidence républicaine : les Bataves au passé, au présent et au futur ». Annales historiques de la Révolution française, 66, 179-194. 25. Willem FRIJHOFF (1990). "L'usage du français en Hollande, XVII ${ }^{\mathrm{e}}$-XIX ${ }^{\mathrm{e}}$ siècles : propositions pour un modèle d'interprétation ". Études de linguistique appliquée, 78, 17-26.

26. Voir par exemple [Justus van EFFEN], De Hollandsche Spectator, $\mathrm{n}^{\circ} 8$ (8 octobre 1731) ; $\mathrm{n}^{\circ}$ 14-15 (19 et 26 novembre 1731); De Philosooph, 17 février 1766. À ce sujet, P.J. BUIJNSTERS (1991). Spectatoriale geschriften. Utrecht: HES, 85-88. L'étonnant dictionnaire juridique publié en 1785 par l'avocat Franciscus Lievens (1785) : Practisyns Woordenboekje. Dordrecht: Abraham Blussé [nouv. éd. par J.E. ENNIK et P. BROOD. Hilversum : Verloren, 1988 ; reprint 2005] montre à quel point le 
langage des tribunaux néerlandais était, outre le latin de procédure, imprégné de mots et expressions français.

27. Nieuwe Spiegel der Jeugd, of Fransche Tiranny (1674). Le Short Title catalogue, Netherlands mentionne des exemplaires à partir de l'impression Amsterdam: Jac. Bouman, 1680. Sur cet ouvrage : E.P. de BOOY (1980). Kweekhoven der wijsheid. Basis- en vervolgonderwijs in de steden van de provincie Utrecht van 1580 tot het begin der $19^{e}$ eeuw. Zutphen: De Walburg Pers, 46, 57; P.J. BUIJNSTERS (1988). « De patriot als schoolmeester: Patriotse ideologie in achttiende-eeuwse kinderboeken ». InTh.S.M. van der ZEE, J.G.M.M. ROSENDAAL \& P.G.B. THISSEN (dir.). 1787 : De Nederlandse Revolutie?Amsterdam : De Bataafsche Leeuw, 100-111. Voir aussi Rotterdam, Atlas van Stolk, no 2551 : 16 gravures sur une feuille volante, publiée par Jac. Bouman à Amsterdam en 1690 . 28. Tiranny der Jacobynsche Factie, in Frankryk (1793). Utrecht, etc. : J. van Rossem, etc.

29. De Fransche tyranny of het moordtoneel, in de jaaren 1792 en 1793 (1793). Amsterdam etc. : Gerrit Bom etc., deux tirages en 1793.

30. S.V.D.T., De Spiegel der jeugd, of Nieuwe Fransche Tiranny (793). Amsterdam: Philippus van Leeuwen.

31. Antoine de COURTIN (1672). Nieuwe verhandeling van de welgemanierdheidt, welke in Vrankryk onder fraaye lieden gebruikelijk is. Amsterdam : J. Blaeu. La version française originale avait aussi été publiée par Jacques LE JEUNE à Amsterdam en 1671.

32. Franciscus RIDDERUS (1674, [ 2 éd. 1738]). Historischen Frans-man, in bysondere Fransche geschiedenissen, gepast op de onderdruckte staat van ons lieve Vaderlandt. Rotterdam : Veuve A. Leers.

33. P.Th.F.M. BOEKHOLT (1991). «Één natie, één taal : taalpolitiek en onderwijshervorming in het begin van de negentiende eeuw ». InK. van BERKEL, H.BOELS \& W.R.H. KOOPS (dir.). Nederland en het Noorden. Assen \& Maastricht: Van Gorcum, 71-80.

34. Gazette nationale, ou Le Moniteur universel, XIII, $\mathrm{n}^{\circ} 268$ (17 juin 1805) [B. N. F : Gr. Fol. Lc2 113, microfilm D 71]. Pour l'impact de cet article aux Pays-Bas : Simon SCHAMA (1997). Patriots and Liberators: Revolution in the Netherlands 1780-1813. New York : Alfred Knopf, 479.

35. Jan ten BRINK (1814). Nieuwe Fransche tirannij, bijzonder onder de regering van Napoleon Bonaparte; behelzende eene opgave van de onregtvaardigheden en geweldenarijen, door de Franschen in Nederland uitgeöefend, sedert het begin van 1795 tot op het einde van 1813, en vooral in de laatste drie jaren. Ten gebruike voor de scholen zamengesteld. Amsterdam: Van der Hey, 88.

\section{RÉSUMÉS}

Dans l'image historique dont la langue française a longtemps bénéficié en Hollande, la thèse de la francisation progressive des élites sous l'Ancien Régime s'est imposée. Tout en reconnaissant l'importance du français dans quelques domaines essentiels de la vie publique, cet article s'efforce de rétablir l'équilibre entre les pratiques et les représentations. Face à la montée du français comme langue de socialisation, langue du commerce international et, remplaçant de plus en plus souvent le latin, comme lingua franca de la science, le néerlandais lui-même s'affirme dès le XVIe siècle comme la langue originelle de l'humanité et la langue privilégiée des arts et des lettres. À la fin du XVIIIe siècle, le français commence à être sérieusement concurrencé par l'allemand et l'anglais dans la littérature et la philosophie, tandis que l'époque révolutionnaire provoque une renaissance $d u$ néerlandais en tant que langue nationale identitaire. 
The historical image of the French language in the Netherlands has privileged the thesis of the on-going 'francization' of the Dutch elites during the Ancient Regime. This article acknowledges the importance of the use of French in several domains of Dutch public life but tries also to reestablish the equilibrium between practices and representation in language use. Undoubtedly, the French language became an important tool for socialisation and commerce, and, next to Latin, it got the statute of lingua franca in the world of science. Yet, the rise of French provoked as early as the 16th century the ambition of Dutch to be recognized as the original language of the humanity and as the privileged language of the arts and letters. At the end of the 18th century, English and German became serious competitors of French in literature and philosophy, whereas the revolutionary period favoured the renaissance of Dutch as the language of national identity.

INDEX

Mots-clés : Pays-Bas. Langue universelle. Langue originelle. Lingua franca. Français. Néerlandais. Allemand. Anglais. Latin. Socialisation. Livres scolaires.

Keywords : Netherlands. Universal language. Original language. Lingua franca. French. Dutch. German. English. Latin. Socialisation. Textbooks.

\section{AUTEUR}

\section{WILLEM FRIJHOFF}

Université Libre, Amsterdam / Université Érasme de Rotterdam 